\title{
Development and Multicentre Validation of the Modena Score to Predict Survival in Advanced Biliary Cancers Undergoing Second-Line Chemotherapy
}

\author{
Massimiliano Salati ${ }^{1,2}$, Luigi Marcheselli ${ }^{1}$, Carlo Messina $\mathbb{D}^{3}{ }^{3}$, Valeria Merz ${ }^{3,4}$, Marco Messina ${ }^{5}$, \\ Pietro Carotenuto $\mathbb{D}^{6}$, Francesco Caputo', Fabio Gelsomino', Andrea Spallanzani', \\ Luca Reggiani Bonetti ${ }^{7}$, Stefania Caramaschi ${ }^{7}$, Gabriele Luppi ', Massimo Dominici (iD)', \\ Michele Ghidini ${ }^{8}$
}

'Division of Oncology, Department of Oncology and Hematology, University Hospital of Modena, Modena, Italy; ${ }^{2}$ PhD Program Clinical and Experimental Medicine, University of Modena and Reggio Emilia, Modena, Italy; ${ }^{3}$ Department of Medical Oncology, Santa Chiara Hospital, Trento, Italy; ${ }^{4}$ Digestive Molecular Clinical Oncology Research Unit, University of Verona, Verona, Italy; ${ }^{5}$ Oncology Unit, Fondazione San Raffaele-Giglio, Cefalu', Palermo, Italy; ${ }^{6}$ Telethon Institute of Genetics and Medicine, Pozzuoli, 80078, Napoli, Italy; ${ }^{7}$ Pathology Section, University Hospital of Modena, Modena, Italy; ${ }^{8}$ Medical Oncology Unit, Fondazione IRCCS Ca' Granda Ospedale Maggiore Policlinico, Milan, Italy

Correspondence: Massimiliano Salati, PhD Program Clinical and Experimental Medicine, University Hospital of Modena, Modena Cancer Centre, via del Pozzo 7I, Modena, 4I I25, Italy, Tel +39/0594223808, Fax +39/0594222647, Email massimiliano.salati@unimore.it

Background: The role of second-line chemotherapy in advanced biliary cancers (ABCs) has only recently been established in phase III randomized trial and the optimal selection of patients most likely to benefit from it remains challenging.

Methods: A cohort of 98 ABC treated second-line chemotherapy was used as a developmental dataset to identify covariates independently associated with overall survival (OS). Kaplan-Meier analysis was used to investigate the association between variables and OS and those retaining statistically significance were combined in a multiplexed score.

Results: The following pretreatment variables were independently associated with OS: ECOG PS $>0$, peritoneal disease, LDH $>430$ $\mathrm{UI} / \mathrm{L}$, albumin $<3.5 \mathrm{gr} / \mathrm{dL}$, gamma-GT $>100 \mathrm{UI} / \mathrm{L}$, sodium $<140 \mathrm{mEq} / \mathrm{L}$, absolute lymphocyte count $<1000 / \mathrm{mmc}$, and PFS to first-line $<6$ months. Based on these results, a scoring system was developed that identified three subgroups with statistically different OS: lowrisk (mOS 18 months), intermediate-risk (mOS 9.4 months) and high-risk (mOS 2.9 months) $(\mathrm{p}<0.001)$. The prognostic model was both internally and externally validated in a multicentre cohort of 120 ABCs.

Conclusion: The Modena score is a multiplexed scoring system capable of accurately risk-stratified ABCs treated with second-line chemotherapy. Based on its reproducibility, usability and generalizability, it has the potential for assisting therapeutic decision-making in the clinic and risk-stratification in future trials.

Keywords: biliary tract cancer, chemotherapy, second-line, prognostic score, survival

\section{Introduction}

Biliary tract cancers (BTCs) are anatomically distinct and genetically heterogeneous tumor entities representing roughly $3 \%$ of gastrointestinal malignancies and the second most common primary liver cancer. Based on their anatomic location within the biliary tree, BTCs are commonly subdivided into intrahepatic (iCCA), perihilar (pCCA) and distal (dCCA) cholangiocarcinoma and gallbladder cancer (GBC). ${ }^{1,2}$ Despite being regarded as relatively rare tumors, their incidence is increasing steadily worldwide, ${ }^{3}$ mainly as a result of a rise in iCCA cases. ${ }^{4-6}$ Surgical resection followed by adjuvant capecitabine offers the only chance of cure in the $15-20 \%$ of patients diagnosed with potentially resectable disease. However, $68-86 \%$ of them still relapse and additional two-thirds present with unresectable advanced-stage disease at 


\section{Graphical Abstract}

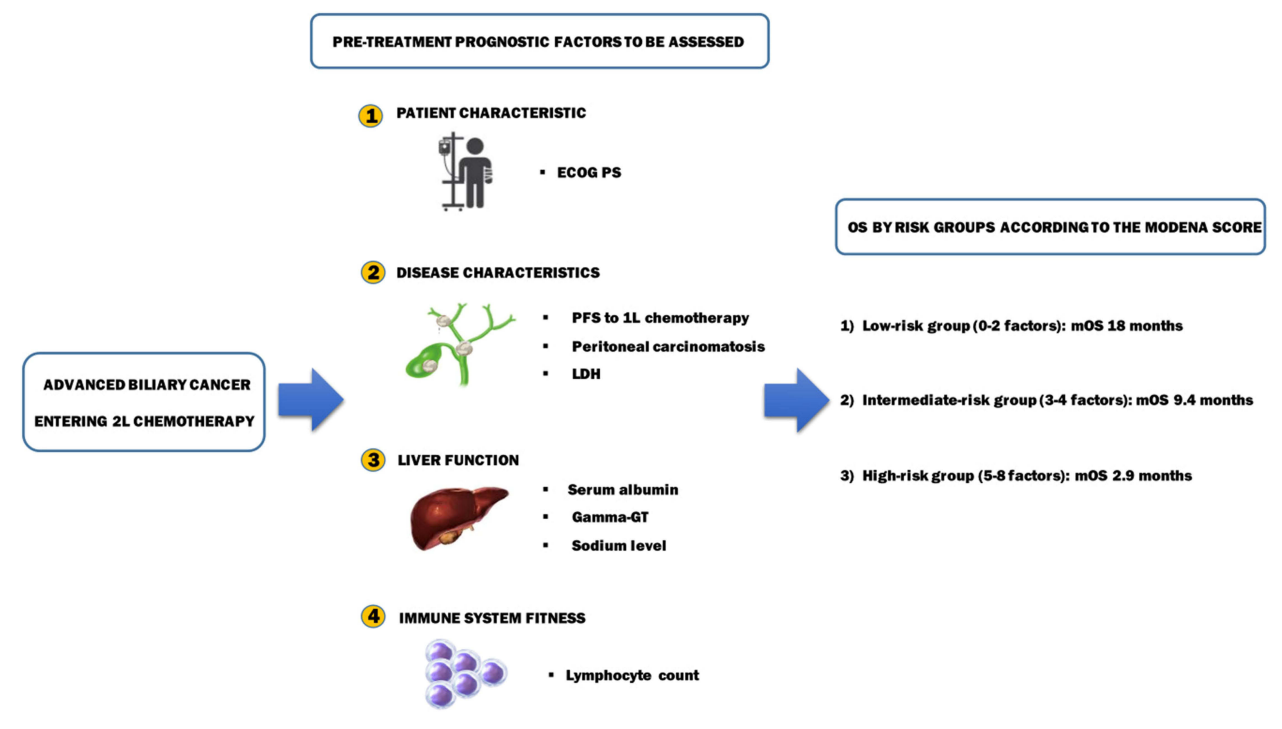

diagnosis. $^{7}$ Palliative-intent chemotherapy in the form of cisplatin and gemcitabine combination is the standard regimen for recurrent, locally advanced and metastatic BTCs resulting in median overall survival (mOS) around 12 months. ${ }^{8}$

For patients failing first-line, until recently only a poor level of evidence (level C) was available to support the use of second-line chemotherapy so that its benefit has been largely debated in recent years. ${ }^{9}$ A systematic review of the literature pooling together data from 25 prospective uncontrolled and retrospective studies involving 761 patients suggested a benefit in selected patients with good performance status (PS). ${ }^{10}$ However, the interpretation of these results has been challenged by potential inherent biases and heterogeneity across studies. In parallel, active symptom control is considered an alternative standard of care endorsed by international guidelines for advanced biliary cancer (ABC) patients who progress following first-line chemotherapy. ${ }^{9}$ Nonetheless, $20-40 \%$ of patients are offered salvage chemotherapy in clinical practice, based on a case-by-case discussion within the multidisciplinary tumor board. ${ }^{11-16}$ Finally, the results of the first prospective randomized phase III trial (ABC-06) in this setting have been presented showing a reduced risk of death for patients treated with mFOLFOX6 regimen compared to active symptom control alone (HR $0.69 ; \mathrm{p}=0.031){ }^{17}$

Notably, the balance between the risks and benefits of a treatment resulting in a 1-month absolute mOS advantage and increased toxicity needs to be carefully evaluated. To this end, there exists an urgent need for factors guiding treatment selection to identify patients more likely to benefit from second-line chemotherapy. In this study, we aimed at developing a user-friendly and robust prognostic tool to assist in the decision-making process of $\mathrm{ABC}$ progressing on or after upfront chemotherapy.

\section{Patients and Methods}

\section{Patient Selection}

Patients with cyto-histologically proven unresectable locally advanced or metastatic BTCs treated with second-line chemotherapy were retrospectively identified from the Modena Cancer Centre Biliary Tract Cancer Database (training cohort; TC).

The validation cohort (VC) was made up of $\mathrm{ABC}$ patients undergoing second-line treatment at the following Italian Centres: Milano, Napoli, Cefalu' (Palermo), Trento and Cremona. 
Patients with mixed hepatocellular-cholangiocellular carcinoma and ampullary carcinoma, as well as those with signs and/or symptoms suggestive of infectious disease within two weeks of starting treatment, were deemed not eligible. Moreover, treatment with immunotherapy, targeted therapy and investigational drug, together with liver-directed locoregional procedures were not allowed.

Baseline clinical and laboratory data were retrieved through electronic medical records review and the following baseline variables were collected and analyzed before the commencement of second-line chemotherapy: age, gender, ECOG PS, primary tumor site, disease status, first-line regimen, progression-free survival (PFS) to first-line. Still, haematological and biochemical parameters including white blood cell count (cell/ $\mu \mathrm{L})$, haemoglobin (gr/dl), platelet count $($ cell $/ \mu \mathrm{L})$, lymphocyte count $($ cell $/ \mu \mathrm{L})$, neutrophil count $($ cell $/ \mu \mathrm{L})$, total bilirubin $(\mathrm{mg} / \mathrm{dL})$, alkaline phosphatase (ALP; IU/L), lactate dehydrogenase (LDH U/L), alanine aminotransferase (ALT; IU/L), aspartate aminotransferase (AST; IU/L), gamma-GT (UI/L), albumin (g/dL), sodium (mEq/L), carbohydrate antigen 19-9 (CA 19-9) (U/mL), and carcinoembryonic antigen $(\mathrm{CEA})(\mathrm{ng} / \mathrm{mL})$.

The study protocol conformed to the ethical guidelines of the 1975 Declaration of Helsinki. Data accessed complying with relevant data protection and privacy regulations were collected under the protocol 183-2019/OSS/AOUMO. The protocol was reviewed and approved by the Area Vasta Emilia Nord Ethics committee, which serves the University Hospital of Modena and the surrounding geographical area.

\section{Statistical Analysis}

The primary endpoint was OS calculated from the date of the first cycle of second-line chemotherapy to the date of death for any cause or last follow-up visit. In descriptive statistics, continuous variables were reported as the median and 2595 percentile, while categorical variables were reported as absolute and percentage frequencies. Laboratory variables initially recorded as continuous parameters were later dichotomized according to the usual clinical thresholds reported in the literature or according to their upper 75 percentile, chosen as worst status. The OS was calculated using KaplanMeier estimators. ${ }^{18}$ Statistical comparisons between the curves were performed with the Log rank test and the effects were estimated using the Cox proportional hazard $(\mathrm{PH})$ regression analysis, with a confidence interval at $95 \%(95 \%$ CI). ${ }^{19}$ The proportionality of hazard was checked graphically using scaled Schoenfeld residuals. ${ }^{20}$ The prognostic performance of each variable on OS was first evaluated through $\mathrm{PH}$ univariate model, selecting covariates with $\mathrm{p}$-value $<0.20$. The final model was developed step by step in multiple Cox PH regression using the likelihood ratio test. Furthermore, the over-optimism and calibration of the model were computed over 250 bootstrap replications employing the Harrell's methods. ${ }^{21}$

The specificity and sensitivity of the prognostic model were evaluated with the ROC-AUC curves according to Heagerty et al. ${ }^{22}$

Both internal and external validations were performed, and measures of model performance were reported to assess the reliability and generalizability of the model. Discrimination is presented graphically by a Kaplan-Meier plot of survival for patients in different risk groups, and the c-Harrell index was used as a measure of discriminant power of the model. Calibration was evaluated through reporting at a fixed time point, a comparison of predicted percentage survival in risk groups with actual survival percentages. ${ }^{23}$

The statistical analyses were performed using Stata version 14.0 or later (StataCorp. LLC, College Station, TX, USA).

\section{Results}

\section{Training Cohort}

Between 1st January 2008 and 31st December 2020, a total of 98 ABC patients (39\%) fulfilling the aforementioned criteria were identified and included in the analysis. The median age of patients was 63 years (range 29-82 years), with a slight prevalence of females (53\%). The most common primary tumor site was iCCA (62\%), followed by eCCA (22\%) and GBC (16\%). All the patients had metastatic disease. 
Overall, the vast majority of patients (72\%) received platinum/gemcitabine doublet chemotherapy as a first-line treatment, resulting in a disease control rate of $69 \%$. The mPFS to first-line was 5 months (range 1-38), with $61 \%$ of patients progressing within 6 months from treatment commencement.

Upon disease progression, fluoropyrimidine-based chemotherapy was the preferred second-line choice in the form of mFOLFIRI (26\%), mFOLFOX6 (20\%) and fluoropyrimidine monotherapy (19\%), respectively. Regarding second-line antitumor activity, the disease control rate was achieved in $39 \%$ of patients, yielding a $7 \%$ of objective responses. Other baseline clinicopathologic and laboratory characteristics are summarized in Table 1.

As of data cut-off, a total of 94 deaths had occurred. The median OS in the whole patient population was 7.2 months (95\% CI; 7-14 months), while the mPFS was 3.5 months; the 1-year OS and 2-year OS were $28 \%$ (95\% CI; 20-37) and $7 \%$ (95\% CI; 3-14), respectively.

\section{Development of the Prognostic Model, Scoring System and Risk Groups}

Globally, among all the covariates tested in the univariate analysis for OS in the VC, the following were associated with shorter OS: male gender $(p=0.032)$, ECOG PS $>0(p<0.001)$, peritoneal involvement $(p<0.001)$, LDH $>430(p=$ $0.001)$, albumin $<3.5 \mathrm{gr} / \mathrm{dl}(\mathrm{p}<0.001)$, absolute lymphocyte count $<1000 / \mathrm{mmc}(\mathrm{p}=0.001)$, absolute monocyte count $>630 / \mathrm{mmc}(\mathrm{p}=0.002)$, absolute neutrophil count $>8000 / \mathrm{mmc}(\mathrm{p}<0.001)$, lymphocyte-monocyte ratio $<2.1(\mathrm{p}=0.001)$, neutrophil-lymphocyte ratio $>3(\mathrm{p}<0.001)$, sodium $<140 \mathrm{mEq} / \mathrm{L}(\mathrm{p}<0.001)$, gamma-G $>100 \mathrm{UI} / \mathrm{L}(\mathrm{p}=0.003)$, GOT $>40 \mathrm{UI} / \mathrm{L}(\mathrm{p}=0.034)$, GPT $>45 \mathrm{UI} / \mathrm{L}(\mathrm{p}=0.005), \mathrm{CA} 19.9>700 \mathrm{UI} / \mathrm{L}(\mathrm{p}<0.001)$, PFS to first-line $<6$ months $(\mathrm{p}<$ 0.001). At the multivariate analysis for OS, the following covariates retained statistical significance as poor prognostic factors: ECOG PS $>0(p=0.002)$, peritoneal involvement $(\mathrm{p}<0.001), \mathrm{LDH}>430 \mathrm{UI} / \mathrm{L}(\mathrm{p}<0.001)$, albumin $<3.5 \mathrm{gr} / \mathrm{dL}$ $(\mathrm{p}=0.001)$, gamma-GT $>100 \mathrm{UI} / \mathrm{L}(\mathrm{p}=0.001)$, sodium $<140 \mathrm{mEq} / \mathrm{L}(\mathrm{p}=0.010)$, absolute lymphocyte count $<1000 / \mathrm{mmc}$ $(p=0.030)$, and PFS to first-line $<6$ months $(p=0.025)$ (Table 2$)$.

Based on these eight variables, a prognostic model was developed to predict OS, and a prognostic score was derived from the model as a sum of the variables, assuming each of these weighted equally to 1 . This scoring system was named after the institution, which serves as the TC (ie Modena Score). Then, three risk groups were identified as follows: a lowrisk group (0-2 negative prognostic factors), an intermediate-risk group (3-4 negative prognostic factors) and a high-risk group (5-8 negative prognostic factors) (Table 3 ). Globally, the patients were evenly distributed across these risk groups, with $27(27 \%)$ patients categorized as low-risk, $35(36 \%)$ patients as intermediate-risk and $36(37 \%)$ as high-risk. The survival was significantly different between groups $(\mathrm{p}<0.001)$ : the mOS for low-, intermediate-, high-risk group was 18 months (95\% CI, 14.7-21-2 months), 9.4 months (95\% CI, 7.9-11.0 months), and 2.9 months (95\% CI, 2.4-3.5 months), respectively. Survival curves according to risk-group stratification are depicted in Figure 1. With regard to the reproducibility of the model, the internal validation showed a shrinkage (overfitting) of 0.128 (250 bootstraps) and c-Harrell index of 0.847 .

The ROC-AUC for the prognostic model in the training sample at 12 months of follow-up was 0.886 , with a specificity of 0.986 and sensitivity of 0.664 at the median value of the linear prediction. The ROC-AUC associated with median linear prediction in the test sample was 0.820 (specificity 0.886 and sensitivity 0.688 ), with an AUC ratio between training/validation sample of 0.926 .

With the aim of providing a simpler and user-friendly pictorial representation of our model, a prognostic nomogram including selected covariates has been constructed (Figure 2).

\section{External Validation Cohort}

The VC included a total of 120 patients (35\%), enrolled from 1st January 2006 to the 31st December 2018 at five Italian Cancer Centres. Baseline demographic and clinicopathologic characteristics were well balanced compared to the TC. The median age was 65 years old, 61 were male (51\%). Overall, 64 (54\%) patients had iCCA, 35 (29\%) GBC and 21 (23\%) eCCA. A total of 78 (65\%) patients received multiagent chemotherapy, among which mFOLFIRI (35\%) was the most commonly adopted regimen, followed by mFOLFOX (29\%). The median OS was 7.5 months ( $95 \%$ CI; $5.9-8.6$ months) and 1-year OS was 17.5\% (95\% CI 10-24). Regarding the prognostic variables of interest: $80(67 \%)$ patients had ECOG PS $>0,30(25 \%)$ had peritoneum involvement, $56(47 \%) \mathrm{LDH}>430 \mathrm{U} / \mathrm{L}, 66(55 \%)$ had albumin $<3.5 \mathrm{~g} / \mathrm{dL}, 30(25 \%)$ 
Table I Baseline Patients' Characteristics in the Training Cohort $(\mathrm{n}=98)$

\begin{tabular}{|c|c|}
\hline Variable & $\mathbf{N}(\%)$ \\
\hline Age, years median & $63(29-82)$ \\
\hline \multicolumn{2}{|l|}{ Gender } \\
\hline Female & $52(53)$ \\
\hline Male & $46(47)$ \\
\hline \multicolumn{2}{|l|}{ ECOG PS } \\
\hline 0 & $62(63)$ \\
\hline $1-2$ & $36(37)$ \\
\hline \multicolumn{2}{|l|}{ Primary tumor site } \\
\hline Intrahepatic cholangiocarcinoma & $61(62)$ \\
\hline Perihilar cholangiocarcinoma & $14(14)$ \\
\hline Distal cholangiocarcinoma & $8(8)$ \\
\hline Gallbladder carcinoma & $15(16)$ \\
\hline \multicolumn{2}{|c|}{ Previous surgery on the primary tumor } \\
\hline Yes & $19(19)$ \\
\hline No & $79(81)$ \\
\hline \multicolumn{2}{|l|}{ Liver metastases } \\
\hline Yes & $85(87)$ \\
\hline No & $13(13)$ \\
\hline \multicolumn{2}{|l|}{ Distant node metastases } \\
\hline Yes & $43(44)$ \\
\hline No & $55(56)$ \\
\hline \multicolumn{2}{|l|}{ Lung metastases } \\
\hline Yes & $28(29)$ \\
\hline No & $70(7 I)$ \\
\hline \multicolumn{2}{|l|}{ Peritoneum metastases } \\
\hline Yes & $26(27)$ \\
\hline No & $72(73)$ \\
\hline \multicolumn{2}{|l|}{ Bone metastases } \\
\hline Yes & $9(9)$ \\
\hline No & $89(91)$ \\
\hline \multicolumn{2}{|l|}{ First-line chemotherapy } \\
\hline Gemcitabine/platinum & $71(72)$ \\
\hline Gemcitabine monotherapy & $15(15)$ \\
\hline
\end{tabular}


Table I (Continued).

\begin{tabular}{|l|c|}
\hline Variable & N (\%) \\
\hline Fluoropyrimidine-based doublet & $12(12)$ \\
\hline Best response to Ist-line & 1 (1) \\
\hline Complete response & $30(31)$ \\
\hline Partial response & $36(37)$ \\
\hline Stable disease & $31(31)$ \\
\hline Progressive disease & \\
\hline 2nd-line chemotherapy & $25(26)$ \\
\hline FOLFIRI & $20(20)$ \\
\hline FOLFOX & $18(18)$ \\
\hline Gemcitabine/platinum & $19(19)$ \\
\hline Fluoropyrimidine monotherapy & $16(16)$ \\
\hline Other &
\end{tabular}

had gamma-GT $>100 \mathrm{UI} / \mathrm{L}, 50(42 \%)$ had progression-free survival to first-line $<6$ months, $42(35 \%) \mathrm{Na}+<140 \mathrm{mEq} / \mathrm{L}$, $71(59 \%)$ absolute lymphocyte count <1000/uL. In the VC, $25(20 \%), 47(40 \%)$ and $48(40 \%)$ patients were assigned to the low-, intermediate- and high-risk group, according to the presence of $0-2,3-4$ and 5-8 risk factors, respectively. The score was able to identify prognostic classes with different median OS: 12.8 months (7.4-11.0), 6.4 months (5.2-8.3) and 2.5 months (1.8-2.7), in the low-, intermediate- and high-risk groups, respectively (p < 0.001) (Figure 3). The performance of the prognostic model in the $\mathrm{VC}$ was adequate, in terms of both discrimination (c-Harrell index 0.712 ; D-Royston 1.467) and prediction accuracy (calibration slope $=0.65$ ).

Table 2 Multiple Cox Proportional Hazard Regression in the Training Cohort $(\mathrm{n}=98)$

\begin{tabular}{|c|c|c|}
\hline Factor & HR (95\% Cl) & p-value \\
\hline ECOG PS $>0$ & $2.33(1.38-3.95)$ & 0.002 \\
\hline Peritoneal involvement & $2.93(1.70-5.07)$ & $<0.001$ \\
\hline LDH $>430$ UI/L & $3.30(1.92-5.67)$ & $<0.001$ \\
\hline Albumin $<3.5 \mathrm{~g} / \mathrm{dL}$ & $3.14(1.55-6.24)$ & 0.001 \\
\hline Gamma-GT > 100 UI/L & $2.44(1.47-4.07)$ & 0.001 \\
\hline $\begin{array}{l}\text { PFS to Ist line }<6 \\
\text { months }\end{array}$ & $1.75(1.07-2.85)$ & 0.025 \\
\hline $\mathrm{Na}+<140 \mathrm{mEq} / \mathrm{L}$ & $2.93(1.29-6.63)$ & 0.010 \\
\hline ALC $<1000 / u L$ & $1.94(1.07-3.53)$ & 0.030 \\
\hline
\end{tabular}


Table 3 Risk-Group Assignment Based on the Prognostic Score in the Training Cohort $(\mathrm{n}=98)$

\begin{tabular}{|l|c|c|c|c|c|}
\hline Risk Group & $\mathbf{N}(\%)$ & I-Year OS\% (95\% CI) & HR (95\% CI) & p-value & Median OS (Months) \\
\hline Low (0/2) & $27(27)$ & $69(48-83)$ & 1.00 & & $18.0(14.7-21.2)$ \\
\hline Intermediate (3-4) & $35(36)$ & $26(13-4 I)$ & $3.78(2.09-6.84)$ & $<0.00 I$ & $9.4(7.9-11.0)$ \\
\hline High (5/8) & $36(37)$ & NA & $32.2(27.0-38.1)$ & $<0.00 I$ & $2.9(2.4-3.5)$ \\
\hline & & High vs Intermediate & $16.5(7.55-25.9)$ & $<0.001$ & \\
\hline
\end{tabular}

Notes: Score: PS, peritoneum, LDH, albumin, ALC, Na+, g-GT, time to Ist progression with weight $=1$. Score from 0 to 8 , stratified in three groups of risk.

\section{Discussion}

In the present study, we developed a prognostic model and a scoring system based on eight variables (ie PFS to first-line, ECOG PS, peritoneal carcinomatosis, LDH, albumin, sodium, lymphocyte count, gamma-GT) showing an accurate performance in risk-stratified $\mathrm{ABC}$ patients treated with second-line chemotherapy into three statistically different prognostic subgroups. Interestingly, we validated our findings in an external independent cohort of 120 patients enrolled across five Italian Cancer Centres.

Only recently, the value of second-line chemotherapy for $\mathrm{ABC}$ has been established in a randomized fashion. ${ }^{17}$ In the phase III ABC-06 trial, 162 patients progressing on or after cisplatin/gemcitabine combination were randomly allocated (81 in each arm) to either active symptom control plus mFOLFOX6 or active symptom control alone. The administration of salvage chemotherapy resulted in improved survival, with mOS and 1-year OS of 6.2 months (vs 5.3 months) and 25.9\% (vs 11.4\%), respectively. As expected, grade 3-4 adverse events, particularly fatigue and neutropenia, were more commonly reported in the experimental arm. On the other hand, new horizons of precision medicine are heading to the targeting of actionable drivers, such as FGFR fusion rearrangements and IDH1/IDH2 mutations with promising results in clinical trials. ${ }^{23-27}$

Although statistically significant, the relative benefit provided by second-line chemotherapy in ABC is clinically marginal, and it has to be balanced against increased toxicity and impaired quality of life. Moreover, only $20 \%$ to $40 \%$ of patients remain candidates to second-line because of their rapidly worsening PS and the occurrence of biliary

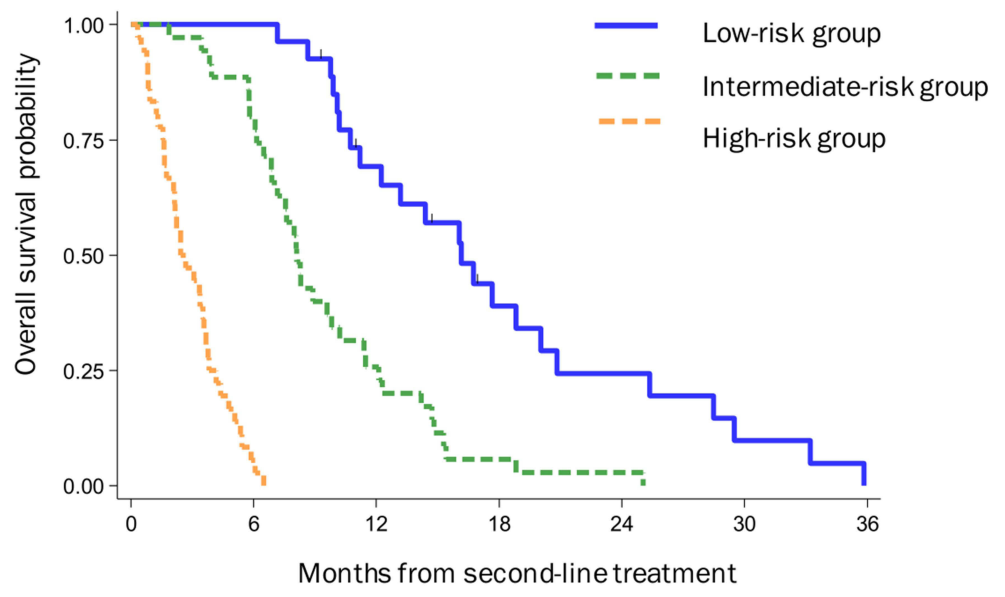

Number at risk

\begin{tabular}{|c|c|c|c|c|c|c|}
\hline High-risk group & 36 & 2 & 0 & 0 & 0 & 0 \\
\hline Intermediate-risk group & 35 & 27 & 8 & 4 & 1 & 0 \\
\hline Low-risk group & 27 & 27 & 18 & 9 & 7 & 1 \\
\hline
\end{tabular}

Figure I Overall survival by risk groups in the training cohort $(n=98)$. Kaplan-Meier curves of overall survival (OS) according to the Modena score in the training cohort. The prognostic score assigned patients to three risk groups with statistically different OS: low-risk ( $\mathrm{n}=27$; mOS 18 months), intermediate-risk ( $\mathrm{n}=35$; mOS 9.4 months) and high-risk ( $\mathrm{n}=27$; mOS 2.9 months) $(\mathrm{p}<0.00 \mathrm{I})$. 
POINTS

\section{1) ECOG PS}

\section{2) Peritoneal involvement}

3) LDH

4) Albumin

5) Gamma-GT

6) PFS to first-line

7) Sodium

8) Absolute lymphocyte count

TOTAL POINTS

6-month Survival Probability

12-month Survival Probability
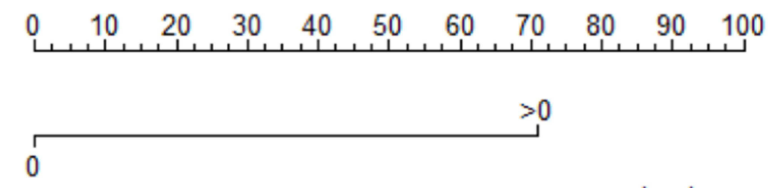

Not inv

Invol.

Not inv.

$$
<=430
$$

$$
\text { 3. }
$$

$3.5+$

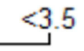

$6+\mathrm{mo}$

$<6 \mathrm{mo}$

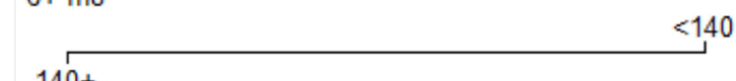

$$
140+
$$
$<1000$

$1000+$

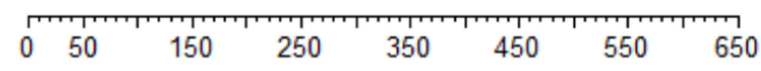

$$
\begin{array}{lllll}
0.9 & 0.80 .7 & 0.5 & 0.250 .1
\end{array}
$$

$$
\begin{array}{lllll}
\hline 0.9 & 0.80 .7 & 0.5 & 0.250 .1
\end{array}
$$

Figure 2 Nomogram for overall survival of biliary cancers treated with second-line chemotherapy. Using and interpreting the nomogram. Each variable incorporated in the Modena score has been listed separately together with the corresponding number of points reflecting its magnitude. For each variable, draw an upward vertical line to the "Points" bar to calculate points, then sum the points for each variable and locate this sum on the "Total Points" axis. Draw a downward vertical line from the "Total Points" line to calculate 6-month and I2-month overall survival probability.

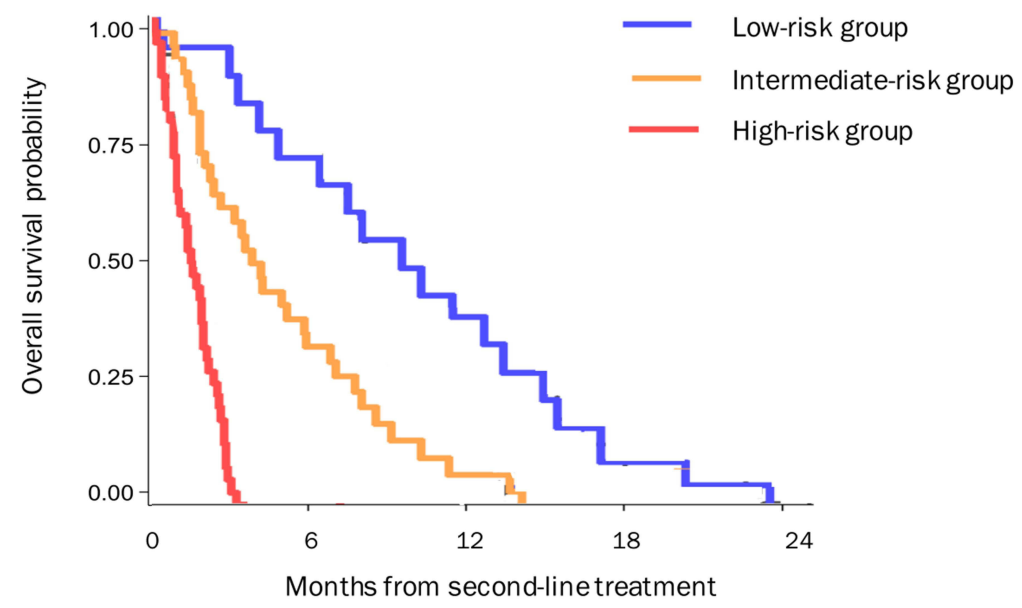

Number at risk

$\begin{array}{lccccc}\text { High-risk group } & 55 & 0 & 0 & 0 & 0 \\ \text { Intermediate-risk group } & 43 & 15 & 3 & 0 & 0 \\ \text { Low-risk group } & 22 & 18 & 11 & 3 & 0\end{array}$

Figure 3 Overall survival by risk groups in the validation cohort $(n=120)$. Kaplan-Meier curves of overall survival (OS) according to the Modena score in the validation cohort. The prognostic score assigned patients to three risk groups with statistically different OS: low-risk ( $n=22 ; \mathrm{mOS} 12.8 \mathrm{months}$ ), intermediate-risk ( $\mathrm{n}=43$; $\mathrm{mOS} 6.4$ months) and high-risk ( $n=55$; mOS 2.5 months) $(p<0.001)$. 
tract-related complications. ${ }^{28-30}$ Nonetheless, no specific molecular signatures or biomarkers have been incorporated in the decision-making process in $\mathrm{ABC}$. Thus, the search for a clinical tool enabling a more accurate prediction of patients' prognosis to maximize the benefit/risk ratio of second-line chemotherapy is an area of active investigation.

Past studies have independently reported several factors to be correlated with survival in this setting, among which are ECOG PS, prior surgery on the primary tumor, disease status, PFS to first-line, and CA19.9. ${ }^{31-36}$ However, individually each factor has limited accuracy in prognostication and is insufficient to reflect the complex biological impact of this disease. More recently, four independent prognostic factors (ECOG PS, the reason for L1 discontinuation, peritoneal carcinomatosis and prior surgery) were combined in a clinical prognostic model developed from a large French retrospective analysis and validated in three multinational patient cohorts $(\mathrm{n}=392) .{ }^{37}$ However, currently, there is neither prospectively validated nor widely accepted clinical prediction model to be routinely applied to the decision-making of ABC undergoing second-line chemotherapy.

The treatment outcomes of our study are comparable to previously published experiences in this setting, showing a mPFS around 3 months and mOS in the range of 6.3-7.2 months. Again, the pattern of care is characterized by a slight prevalence of irinotecan-based doublets as consistently reported across Europe, except in the UK, where oxaliplatinbased regimens are more common following cisplatin/gemcitabine first-line.

Of note, we were able to confirm the prognostic value of established clinical variables in the multivariate analysis, including ECOG PS, peritoneal carcinomatosis and PFS to the first-line. More interestingly, we provided a comprehensive characterization of potential prognosticators, including in our analysis additional biological factors compared to previous studies. To this end, we showed for the first time that LDH, sodium, lymphocyte count, and gamma-GT were independent predictors of outcome in this setting. In particular, the inclusion of liver function parameters represents an added value in light of the sizeable proportion of iCCA $(>50 \%)$ recruited in the study, among whom chronic liver disease is a highly prevalent underlying condition.

A multiplexed score incorporating patient (ECOG PS), disease (PFS to first-line, peritoneal carcinomatosis, LDH), organ function (albumin, sodium, gamma-GT) and immune system (lymphocyte count) determinants may better account for the heterogeneity and complexity of this disease. Hence, offering a more accurate tool for prognosis prediction for both trial design and daily practice. When applied to the clinical scenario, the Modena score may identify a subgroup of $\mathrm{ABC}$ portending a particularly poor prognosis (OS $<3$ months), for whom best supportive care alone seems advisable. On the other hand, the intermediate- and even more the low-risk group seem to be the best candidates for a treatment strategy with multiple lines in light of their favourable behaviour. The retrospective design of our study along with its relatively small sample size are limitations to be acknowledged that may have precluded us from the proper assessment of additional variables of potential interest as well from the presence of confounders. Still, prospective validation of our findings in larger patient populations is required in the future.

\section{Conclusions}

In conclusion, we provided evidence of a clinical prognostic model and score constructed in a rigorous methodological framework for $\mathrm{ABC}$ amenable to second-line chemotherapy. This model takes into account several prognostic determinants and bears the potential for improving the accuracy of currently available prediction tools. It is proved to be reproducible, easily usable, and generalizable. We, therefore, believe that it could become a useful tool for guiding therapeutic decisions in clinical practice and risk-stratification in future clinical trials.

\section{Author Contributions}

All authors contributed to data analysis, drafting or revising the article, have agreed on the journal to which the article will be submitted, gave final approval for the version to be published, and agree to be accountable for all aspects of the work.

\section{Funding}

There is no funding to report. 


\section{Disclosure}

Dr Luigi Marcheselli reports scientific consultant for Sandoz Spa during Jan 2021-Sep 2022, free of fee. Dr Fabio Gelsomino reports personal fees from Servier, Iqvia, Eli Lilly, Merck Serono, and Amgen, outside the submitted work. The authors report no other conflicts of interest in this work.

\section{References}

1. Razumilava N, Gores GJ. Cholangiocarcinoma. Lancet. 2014;383:2168-2179. doi:10.1016/S0140-6736(13)61903-0

2. Braconi C, Patel T. Cholangiocarcinoma: new insights into disease pathogenesis and biology. Infect Dis Clin North Am. 2010;24(4):871-84, vii. doi:10.1016/j.idc.2010.07.006

3. Bray F, Ferlay J, Soerjomataram I, Siegel RL, Torre LA, Jemal A. Global cancer statistics 2018: GLOBOCAN estimates of incidence and mortality worldwide for 36 cancers in 185 countries. CA Cancer J Clin. 2018;68:394-424. doi:10.3322/caac.21492

4. Patel T. Increasing incidence and mortality of primary intrahepatic cholangiocarcinoma in the United States. Hepatology. 2001;33:1353-1357. doi:10.1053/jhep.2001.25087

5. Saha SK, Zhu AX, Fuchs CS, Brooks GA. Forty-year trends in cholangiocarcinoma incidence in the US: intrahepatic disease on the rise. Oncologist. 2016;21:594-599. doi:10.1634/theoncologist.2015-0446

6. Welzel TM, McGlynn KA, Hsing AW, O'Brien TR, Pfeiffer RM. Impact of classification of hilarcholangiocarcinomas (Klatskin tumors) on the incidence of intra- and extrahepatic cholangiocarcinoma in the United States. $J$ Natl Cancer Inst. 2006;98:873-875. doi:10.1093/jnci/djj234

7. Adeva J, Sangro B, Salati M, et al. Medical treatment for cholangiocarcinoma. Liver Int. 2019;39:123-142. doi:10.1111/liv.14100

8. Valle J, Wasan H, Palmer DH, et al. Cisplatin plus gemcitabine versus gemcitabine for biliary tract cancer. N Engl J Med. 2010;362:1273-1281. doi:10.1056/NEJMoa0908721

9. Valle J, Borbath I, Khan SA, Huguet F, Gruenberger T, Arnold D. Biliary Cancer: ESMO clinical practice guidelines. Ann Oncol. 2016;27(suppl 5): v28-v37. doi:10.1093/annonc/mdw324

10. Lamarca A, Hubner RA, David Ryder W, Valle J. Second-line chemotherapy in advanced biliary cancer: a systematic review. Ann Oncol. 2014;5 (12):2328-2338. doi:10.1093/annonc/mdu162

11. Salati M, Caputo F, Cunningham D, et al. The A.L.A.N. score identifies prognostic classes in advanced biliary cancer patients receiving first-line chemotherapy. Eur J Cancer. 2019;117:84-90. doi:10.1016/j.ejca.2019.05.030

12. Bridgewater J, Lopes A, Wasan H, et al. Prognostic factors for progression-free and overall survival in advanced biliary tract cancer. Ann Oncol. 2016;27:134-140. doi:10.1093/annonc/mdv483

13. McNamara MG, Templeton AJ, Maganti M, et al. Neutrophil/lymphocyte ratio as a prognostic factor in biliary tract cancer. Eur J Cancer. 2014;50:1581-1589. doi:10.1016/j.ejca.2014.02.015

14. Peixoto RD, Renouf D, Lim H. A population based analysis of prognostic factors in advanced biliary tract cancer. J Gastrointest Oncol. 2014;5:428-432. doi:10.3978/j.issn.2078-6891.2014.081

15. Suzuki Y, Kan M, Kimura G, et al. Predictive factors of the treatment outcome in patients with advanced biliary tract cancer receiving gemcitabine plus cisplatin as first-line chemotherapy. $J$ Gastroenterol. 2019;54:281-290. doi:10.1007/s00535-018-1518-3

16. Salati M, Caputo F, Cerma K, Marcheselli L, Braconi C, Cascinu S. The impact of gender and immune system determinants on long-term survival in biliary tract cancer. Ital J Gend Specif Med. 2019;5:27-30. doi:10.1723/3148.31296

17. Lamarca A, Palmer DH, Wasan HS, et al. Second-line FOLFOX chemotherapy versus active symptom control for advanced biliary tract cancer (ABC-06): a Phase 3, open-label, randomised, controlled trial. Lancet Oncol. 2021;22(5):690-701. PMCID: PMC8082275. doi:10.1016/S14702045(21)00027-9

18. Lamarca A, Palmer DH, Wasan HS, et al. ABC-06: a randomised Phase III, multi-centre, open-label study of active symptom control (ASC) alone or ASC with oxaliplatin/5-FU chemotherapy (ASC+mFOLFOX) for patients (pts) with locally advanced/metastatic biliary tract cancers (ABC) previously-treated with cisplatin/gemcitabine (CisGem) chemotherapy. Proc Am Soc Clin Oncol. 2019;37:Article 4003.

19. Kaplan EL, Meier P. Non parametric estimation from incomplete observations. J Am Stat Assoc. 1958;53:457-81. doi:10.1080/ 01621459.1958 .10501452

20. Cox D. Regression models and life tables. J R Stat Soc. 1972;34:187-202. Series B.

21. Schoenfeld D. Partial residuals for proportional hazard regression model. Biometrika. 1982;69:239-41. doi:10.1093/biomet/69.1.239

22. Heagerty PJ, Lumley T, Pepe MS. Time-dependent ROC curves for censored survival data and a diagnostic marker. Biometrics. 2000;56:337-344. doi:10.1111/j.0006-341X.2000.00337.x

23. Harrel FE, Lee HL, Mark DB. Multivariable prognostic model: issues in developing model. Evaluating assumptions and adequacy and measuring and reducing errors. Stat Med. 1996;15:361-87.

24. Javle M, Lowery M, Shroff RT, et al. Phase II study of BGJ398 in patients with FGFR-altered advanced cholangiocarcinoma. J Clin Oncol. 2018;36:276-282. doi:10.1200/JCO.2017.75.5009

25. Meric-Bernstam F, Arkenau H, Tran B, et al. Efficacy of TAS-120, an irreversible fibroblast growth factor receptor (FGFR) inhibitor, in cholangiocarcinoma patients with FGFR pathway alterations who were previously treated with chemotherapy and other FGFR inhibitors. Ann Oncol. 2018;29:ix46-ix66. doi:10.1093/annonc/mdy149

26. Abou-Alfa GK, Sahai V, Hollebecque A, et al. Pemigatinib for previously treated, locally advanced or metastatic cholangiocarcinoma: a multicentre, open-label, Phase 2 study. Lancet Oncol. 2020;21:671-684. doi:10.1016/S1470-2045(20)30109-1

27. Mazzaferro V, El-Rayes BF, Droz Dit Busset M, et al. Derazantinib (ARQ 087) in advanced or inoperable FGFR2 gene fusion-positive intrahepatic cholangiocarcinoma. Br J Cancer. 2019;120:165-171. doi:10.1038/s41416-018-0334-0

28. Abou-Alfa GK, Macarulla Mercade T, Javle M, et al. ClarIDHy: a global, phase III, randomized, double-blind study of ivosidenib (IVO) vs placebo in patients with advanced cholangiocarcinoma (CC) with an isocitrate dehydrogenase 1 (IDH1) mutation. Ann Oncol. 2019;30(mdz394.027.):v872v873. doi:10.1093/annonc/mdz394.027

29. Abou-Alfa GK, Macarulla T, Javle MM, et al. Ivosidenib in IDH1-mutant, chemotherapy-refractory cholangiocarcinoma (ClarIDHy): a multicentre, randomised, double-blind, placebo-controlled, phase 3 study. Lancet Oncol. 2020;21:796-807. doi:10.1016/S1470-2045(20)30157-1 
30. Salati M, Braconi C. Noncoding RNA in Cholangiocarcinoma. Semin Liver Dis. 2019;39:013-025. doi:10.1055/s-0038-1676097

31. Carotenuto P, Hedayat S, Fassan M, et al. Modulation of biliary cancer chemo-resistance through microRNA-mediated rewiring of the expansion of CD133+ cells. Hepatology. 2019. doi:10.1002/hep.31094

32. Salati M, Filippi R, Vivaldi C, et al. The prognostic nutritional index predicts survival and response to first-line chemotherapy in advanced biliary cancer. Liver Int. 2019. doi:10.1111/liv.14314

33. Lowery MA, Goff LW, Keenan BP, et al. Second-line chemotherapy in advanced biliary cancers: a retrospective, multicenter analysis of outcomes. Cancer. 2019;125(24):4426-4434. doi:10.1002/cncr.32463

34. Fornaro L, Cereda S, Aprile G, et al. Multivariate prognostic factors analysis for second-line chemotherapy in advanced biliary tract cancer. $B r$ $J$ Cancer. 2014;110:2165-2169. doi:10.1038/bjc.2014.190

35. Walter T, Horgan AM, McNamara M, et al. Feasibility and benefits of second-line chemotherapy in advanced biliary tract cancer: a large retrospective study. Eur J Cancer. 2013;49(2):329-335. doi:10.1016/j.ejca.2012.08.003

36. Brieau B, Dahan L, De Rycke Y, et al. Second-line chemotherapy for advanced biliary tract cancer after failure of the gemcitabine-platinum combination: a large multicenter study by the Association des Gastro-Entérologues Oncologues. Cancer. 2015;121(18):3290-3297. doi:10.1002/ cncr.29471

37. Neuzillet C, Casadei Gardini A, Brieau B, et al. Prediction of survival with second-line therapy in biliary tract cancer: actualisation of the AGEO CT2BIL cohort and European multicentre validations. Eur J Cancer. 2019;111:94-106. doi:10.1016/j.ejca.2019.01.019

\section{Publish your work in this journal}

Cancer Management and Research is an international, peer-reviewed open access journal focusing on cancer research and the optimal use of preventative and integrated treatment interventions to achieve improved outcomes, enhanced survival and quality of life for the cancer patient. The manuscript management system is completely online and includes a very quick and fair peer-review system, which is all easy to use. Visit http://www.dovepress.com/testimonials.php to read real quotes from published authors.

Submit your manuscript here: https://www.dovepress.com/cancer-management-and-research-journal 